\title{
Distal radial cortical bone thickness correlates with bone mineral density and can predict osteoporosis: a cohort study
}

\section{ChunXiao Ye}

Quanzhou Orthopedic-Traumatological Hospital

YingBin Guo ( $\nabla$ yingbinguo@hotmail.com )

YouHui Zheng

Quanzhou Orthopedic-Traumatological Hospital

\section{ZhenBin Wu}

Quanzhou Orthopedic-Traumatological Hospital

KaiYu Chen

Quanzhou Orthopedic-Traumatological Hospital

\section{XiaoLing Zhang}

Quanzhou Orthopedic-Traumatological Hospital

\section{LiangXiu Xiao}

Luoyang Orthopedic-Traumatological Hospital

\section{ZhiMing Chen}

Quanzhou Orthopedic-Traumatological Hospital

\section{Research article}

Keywords: Distal radius fracture; Osteoporosis; Cortical thickness; Bone mineral density; Fragility fracture

Posted Date: February 27th, 2020

DOI: https://doi.org/10.21203/rs.2.24768/v1

License: (9) This work is licensed under a Creative Commons Attribution 4.0 International License. Read Full License

Version of Record: A version of this preprint was published on August 9th, 2020. See the published version at https://doi.org/10.1016/j.injury.2020.08.018. 


\section{Abstract}

Background We evaluated the correlation of the bone mineral density (BMD) of the hip and lumbar spine with the distal radius cortical thickness (DRCT) measured on anteroposterior radiographs to establish a method for predicting osteoporosis. Methods We assessed 147 patients aged $\geq 50$ years with distal radius fractures who underwent wrist radiographs and dual-energy X-ray absorptiometry. The DRCT was measured and calculated at two levels of the distal radius of the injured wrist on the radiographs. Results In the Pearson correlation and simple linear regression analyses, the DRCT was positively correlated with hip BMD $(r=0.393, P<0.01)$ and lumbar spine BMD $(r=0.529, P<0.01)$. Each 1-mm increase in DRCT was associated with a $0.051-\mathrm{g} / \mathrm{cm} 2$ increase in hip BMD $(R 2=0.154, P<0.01)$ and a $0.080-\mathrm{g} / \mathrm{cm} 2$ increase in lumbar spine BMD ( $2=0.280, P<0.01)$. A DRCT of $5.1 \mathrm{~mm}$ was selected as the cutoff point for predicting osteoporosis, with the highest Youden index of $0.560,83.3 \%$ sensitivity, $72.7 \%$ specificity, and a $66.7 \%$ negative predictive value. Conclusion Cortical thickness measurements obtained from anteroposterior wrist radiographs were positively correlated with hip and lumbar spine BMD measurements. This technique is suggested as a rapid, inexpensive, and sensitive method for predicting osteoporosis.

\section{Background}

A fracture of the distal part of the radius is one of the most common types of fracture, and its incidence increases with age, particularly in female patients [1]. Such fractures have been shown to be associated with osteoporosis [1-3].

Patients with distal radius fractures have a 1.4- to 2.7-fold increased risk of secondary hip fracture [1, 2]. Studies have demonstrated that a high proportion of patients with distal radius fractures have low bone mineral density (BMD) [4-6], indicating that this type of fracture might be the first manifestation of osteoporosis. Patients with low-energy distal radius fractures are more likely to sustain secondary hip and spinal fractures [4, 7]. The gold standard for diagnosing osteoporosis is dual-energy X-ray absorptiometry (DXA) [8]. However, a large number of patients with fractures related to osteoporosis are not evaluated and treated for osteoporosis [9-11].

A sensitive and rapid method with which to evaluate osteoporosis would be helpful to clinicians who have questions about patients' bone quality but have no immediate access to DXA. Such a method could also be helpful for preliminary assessment of osteoporosis.

In 1960, Barnett and Nordin [12] measured the thickness of the metacarpal cortex and studied the relationship between the cortical bone thickness and BMD. Several researchers have measured the cortical thickness at different anatomical sites [13]. Tingart et al. [14] performed a cadaveric study and found that the cortical thickness of the proximal humerus measured on radiographs was correlated with that measured by DXA. More recently, Mather et al. [15] indicated that the proximal humeral cortical thickness was positively correlated with the hip and lumbar spine BMD measured by DXA. Tracy et al. [16] 
demonstrated that the distal radius cortical thickness (DRCT) was positively correlated with the hip BMD measured by DXA, but not with the lumbar spine BMD measured by DXA.

The present study was performed to evaluate the correlation of the BMD of the hip and lumbar spine with the DRCT measured on anteroposterior radiographs. Our goal was to establish a method for predicting osteoporosis by measuring the DRCT on radiographs.

\section{Methods}

\subsection{Patients}

From May 2015 to November 2017, the BMD of the hip and lumbar spine was measured in 147 patients aged $\geq 50$ years with a radiographic diagnosis of distal radius fractures in the Upper Extremity Service of our hospital. The patients were divided into a non-osteoporotic group and osteoporotic group according to the T value of DXA. The non-osteoporotic group comprised 51 patients ( 4 men, 47 women) with a mean age of $58.2 \pm 6.4$ years (range, $50-76$ years), mean body weight of $62.4 \pm 8.8 \mathrm{~kg}$ (range, $42.5-$ $82 \mathrm{~kg}$ ), and mean body mass index (BMI) of $25.4 \pm 3.5 \mathrm{~kg} / \mathrm{m}^{2}$. The osteoporotic group comprised 96 patients ( 9 men, 87 women) with a mean age of $66.8 \pm 9.4$ years (range, 52-88 years), mean body weight of $55.8 \pm 8.1 \mathrm{~kg}$ (range, $40-88 \mathrm{~kg}$ ), and mean BMl of $23.4 \pm 4.0 \mathrm{~kg} / \mathrm{m}^{2}$ ]

\subsection{Measurement of BMD}

The BMD of the lumbar spine (L1-4) and total hip was measured using a DXA scanner (Discovery Wi; Hologic Inc., Waltham, MA, USA). The patients were referred for DXA within 7 days (mean, $2.8 \pm 1.2$ days) after fracture.

\subsection{Measurement of DRCT}

The DRCT measurement technique is shown in Fig. 1. We used a self-contained program to make all radiographic measurements from a Picture Archiving and Communications System workstation (Lanyun Medical Imaging Co., Ltd., Shenzhen, China). We found that the medial and lateral cortex of the distal radius became parallel at about $40 \mathrm{~mm}$ proximal to the distal radial ulnar joint on the posteroanterior radiographs. The measurement method we used was based on the study by Tracy et al. [16]. Line A was drawn parallel to the radial medullary canal, and Line B was drawn passing through the distal ulnar articular plane and perpendicular to Line A. The first measurement point was $40 \mathrm{~mm}$ away from the straight Line B. We then drew Line M1, which passed through the measurement point and outer cortex of the radius and was parallel to Line $B$. Line $M 2$ was drawn at the same level and extended to the inner cortex of the radius. The level of cortical thickness $\mathrm{C} 1$ was defined as M1 - M2. This process was repeated to measure $M 3, M 4$, and $C 2$ at the second measurement point, which was $60 \mathrm{~mm}$ away from the straight Line B. The DRCT was defined as $(\mathrm{C} 1+\mathrm{C} 2) / 2$, and the cortex ratio was defined as $2 \mathrm{DRCT} /(\mathrm{M} 1+$ M3). The measurements were performed twice by two different doctors at different times to determine the intra- and inter-observer variability. 


\subsection{Statistical analysis}

SPSS 16.0 software (SPSS Inc., Chicago, IL, USA) was used for statistical analysis of the data.

Measurement data are presented as mean \pm standard deviation, and the $t$ test was applied to all outcome analyses where appropriate. Two continuous random variables were analyzed by Pearson's correlation analysis with unitary linear regression.

The sensitivity, specificity, negative predictive value (NPV), and Youden index of the DRCT threshold values for predicting osteoporosis were calculated using the World Health Organization classification system for osteoporosis. For all tests, a $\mathrm{P}$ value of $<0.05$ was considered statistically significant.

\section{Results}

\subsection{Subgroup analysis}

The 96 patients in the osteoporotic group ( 9 men, 87 women) had a mean DRCT of $4.22 \pm 1.01 \mathrm{~mm}$ and mean cortex ratio of $0.29 \pm 0.08$. The 51 patients in the non-osteoporotic group ( 4 men, 47 women) had a mean DRCT of $5.33 \pm 0.85 \mathrm{~mm}$ and mean cortex ratio of $0.35 \pm 0.07$. Patients in the osteoporotic group were older $(t=6.584, P=0.000)$, had a lower body weight $(t=-4.517, P=0.000)$, had a thinner DRCT $(t=$ $-7.009, P=0.000)$, had a lower cortex ratio $(t=-4.548, P=0.000)$, and had a lower $B M I(t=-2.991, P=$ $0.003)$ than patients in the non-osteoporotic group. The patients' characteristics in the two groups are displayed in Table 1.

\subsection{Correlations among DRCT, cortex ratio, and BMD}

For the entire cohort, the mean DRCT was $4.60 \pm 1.09 \mathrm{~mm}$, the mean cortex ratio was $0.309 \pm 0.078$, the mean hip BMD was $0.67 \pm 0.14 \mathrm{~g} / \mathrm{cm}^{2}$, and the mean lumbar spine BMD was $0.71 \pm 0.16 \mathrm{~g} / \mathrm{cm}^{2}$. Linear regression analysis showed that DRCT was positively correlated with hip BMD $(r=0.393, P=0.000)$ and lumbar spine BMD $(r=0.529, P=0.000)$ and that the cortex ratio was positively correlated with hip BMD $(r=0.337, P=0.000)$ and lumbar spine BMD $(r=0.414, P=0.000)$. Each 1-mm increase in DRCT was associated with a $0.051-\mathrm{g} / \mathrm{cm}^{2}$ increase in hip BMD $\left(R^{2}=0.154, P=0.000\right)$ and a $0.080-\mathrm{g} / \mathrm{cm}^{2}$ increase in lumbar spine $B M D\left(R^{2}=0.280, P=0.000\right)$. Each 1-unit increase in the cortex ratio was associated with a $0.617-\mathrm{g} / \mathrm{cm}^{2}$ increase in hip BMD $\left(R^{2}=0.114, P=0.000\right)$ and a $0.870-\mathrm{g} / \mathrm{cm}^{2}$ increase in lumbar spine $\mathrm{BMD}\left(\mathrm{R}^{2}=0.171, \mathrm{P}=0.000\right)$ (Table 2, Figs. $\left.2-5\right)$.

\subsection{Predictive value of DRCT in determining osteoporosis}

The sensitivity, specificity, NPV, and Youden index of the DRCT threshold values for predicting osteoporosis were calculated. A cutoff point of $5.5 \mathrm{~mm}$ showed $92.7 \%$ sensitivity, $43.1 \%$ specificity, and a 74.1\% NPV (Youden index, 0.358 ). A cutoff point of $5.4 \mathrm{~mm}$ showed $91.7 \%$ sensitivity, $51.0 \%$ specificity, and a 76.5\% NPV (Youden index, 0.427). A cutoff point of $5.3 \mathrm{~mm}$ showed $90.6 \%$ sensitivity, $51.0 \%$ specificity, and a $74.3 \%$ NPV (Youden index, 0.416 ). A cutoff point of $5.2 \mathrm{~mm}$ showed $88.5 \%$ sensitivity, 
$58.8 \%$ specificity, and a $63.2 \%$ NPV (Youden index, 0.473). A cutoff point of $5.1 \mathrm{~mm}$ showed $83.3 \%$ sensitivity, $72.7 \%$ specificity, and a 66.7\% NPV (Youden index, 0.560). Finally, a cutoff point of $5.0 \mathrm{~mm}$ showed $78.1 \%$ sensitivity, $56.1 \%$ specificity, and a 52.3\% NPV (Youden index, 0.342). Accordingly, $5.1 \mathrm{~mm}$ was selected as the best diagnostic cutoff point for osteoporosis (Table 3 ). 


\section{Discussion}

Osteoporosis is a common disease in the older population, and the incidence of osteoporosis increases with age. In one study, the incidence of femoral osteoporosis increased from $5 \%$ in 50 to 59 -year-old women to $66 \%$ in 80 - to 89 -year-old women [17]. An osteoporotic fracture is a clear manifestation of decreased bone strength and is the ultimate result of osteoporosis $[18,19]$. Many studies have indicated that each standard deviation reduction in BMD results in a 1.4- to 2.6-fold increase in fracture risk [20]. Soichiro and Hideki [21] found that BMD began to decline at the age of 50 years and continued to decline rapidly until the mid-60 s, then gradually declined until the age of 90 years.

In the present study, both the BMD and DRCT of the entire cohort decreased with age (Fig. 6). Because of the small number of patients, we were unable to analyze the rate of BMD decline among different age groups. However, patients with osteoporosis were significantly older than those without osteoporosis ( $t=$ $6.584, P=0.000)$, indicating that older age is associated with osteoporosis.

Observational studies have shown that both body weight and $\mathrm{BMI}$ are modifiable factors affecting BMD $[22,23]$. Obese people have a lower risk of forearm, hip, and pelvis fractures [24].

Studies have shown that a higher body weight places greater mechanical stress on the bones, and this stress is a direct stimulator of bone formation [25]. Patients categorized as osteoporotic had significantly lower body weight $(t=-4.517, \mathrm{P}=$ $0.000)$, suggesting that a higher weight-related load is associated with higher bone mass.

Rausch et al. [26] found a correlation between the thickness of the radial cortex and the radial BMD in a study of 12 advancedage cadavers. Ward et al. [27] found that the cortical bone thickness decreased by $14 \%$ every 10 years after 50 years of age.

Pistoia et al. [28] reported that decreases in the cortical bone thickness, trabecular thickness, and trabecular number can cause a decrease in bone strength, and the decrease in the cortical bone thickness had the most pronounce effect on the decrease in bone strength. We performed the present study to determine the relationship between the DRCT and BMD of the hip and lumbar spine and assess the ability of the DRCT to predict osteoporosis. We found that the mean DRCT was thinner in the osteoporotic than non-osteoporotic group $(t=-7.009, P=0.000)$, indicating that a thinner bone cortex is associated with osteoporosis. This is consistent with previous studies.

Although we found that the DRCT was positively correlated with the hip and lumbar BMD, there was no correlation between the DRCT and lumbar spine BMD in the study by Tracy et al. [16].

They reported that the axial skeleton differs from the appendicular skeleton in loading properties and distribution of bearing stress and that the BMD changes with aging in different ways.

Of the two techniques assessed in our study, the DRCT had a stronger correlation with the hip DXA measurement than the 
cortex ratio $\left(R^{2}=0.154\right.$ vs. 0.114$)$ and a stronger correlation with the lumbar spine DXA measurement $\left(R^{2}=0.280\right.$ vs. 0.171$)$, suggesting that the DRCT is a better measurement parameter than the cortex ratio for predicting osteoporosis. We selected a

DRCT of $5.1 \mathrm{~mm}$ as the best diagnostic cutoff point for the evaluation of osteoporosis. This indicates that a patient whose DRCT is $<5.1 \mathrm{~mm}$ may be required to undergo further DXA testing to determine the presence of osteoporosis and that such patients could benefit from DXA testing and osteoporosis treatment.

In the study by Tracy et al. [16], all patients were female and most were Caucasian, and the cutoff point for diagnosis was

$5.5 \mathrm{~mm}$. However, no NPV was provided. Another study

performed by Mather et al. [15] showed that the bicortical thickness and cortex ratio of the proximal humerus were much more strongly correlated with the DXA measurement of the femur than the lumbar spine. This suggests that although the cortical thickness may differ among different races, between the two sexes, and even among multiple anatomic sites, it seems to be associated with BMD.

DXA cannot be replaced by measurement of the cortical thickness, which may be an early predictor of osteoporosis. Hollevoe and Verdonk [29] noted that the correlation between the $\mathrm{BMD}$ and the clinical results of distal radius fractures was much stronger than the correlation between the BMD and cortical thickness and suggested that BMD can predict the clinical prognosis. X-ray examination is routinely used for assessment of musculoskeletal system diseases, especially hand and wrist injuries. Patients with a thinning bone cortex should be recommended to undergo further DXA examination to rule out osteoporosis. Because many patients refuse to undergo DXA and follow-up [30], measurement of the cortical thickness may provide an opportunity to predict the risk of further fragility fractures, and the incidence of debilitating fractures can then be reduced by early balance intervention, fall prevention, and osteoporosis treatment.

Limitations of this study include the relatively small sample size and the lack of consideration of the influence of group, age, weight, and some other factors. The results of this study are only applicable to patients aged $\geq 50$ years and need to be confirmed in a randomized controlled trial with a larger sample size. This will allow the interaction among the factors that affect BMD to be explained and evaluated comprehensively and reasonably.

\section{Conclusion}

X-ray measurement of the DRCT was positively correlated with hip and lumbar BMD. The technique described herein is suggested as an effective, inexpensive, and rapid method for differentiation of osteoporosis.

\section{List Of Abbreviations}

BMD: bone mineral density 
DXA: dual-energy X-ray absorptiometry

DRCT: distal radial cortical thickness

BMI: body mass index

NPV: negative predictive value

\section{Declarations}

\section{Ethics approval and consent to participate}

This study was approved by the Quanzhou OrthopedicTraumatological Hospital Medical Ethics Committee\Approval No.QZOTEC-2019-005, Quanzhou Orthopedic-Traumatological

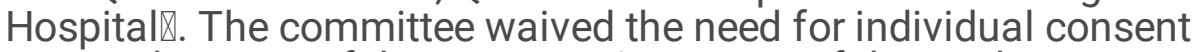
because of the retrospective nature of the study.

\section{Consent to publish}

The patients provided written informed consent for publication of their clinical details and clinical images. A copy of the consent form is available for review by the Editor of this journal.

\section{Availability of data and materials}

All data generated or analysed during this study are included in this published article.

\section{Competing interests}

The authors declare that they have no competing interests.

\section{Funding}

This work was sponsored by a fund from the Quanzhou Science and Technology Project (2019N002S). The funding source had

no role in the design of the study; collection, analysis, and interpretation of data; and in writing the manuscript.

\section{Authors' contributions}

CX designed the study, collected the data, analyzed the data, carried out the statistical analysis, and wrote the manuscript. LX,

$\mathrm{ZM}$, and $\mathrm{XL}$ collected the data. $\mathrm{ZB}, \mathrm{YH}$, and $\mathrm{KY}$ collected and analyzed the data. YB participated in the design and

coordination of the study. All authors read and approved the final manuscript.

\section{Acknowledgment}

We thank Angela Morben, DVM, ELS, from Liwen Bianji, Edanz Editing China (www.liwenbianji.cn/ac), for editing the English text of a draft of this manuscript.

\section{References}


[1] Klotzbuecher CM, Ross PD, Landsman PB, et al. Patients with prior fractures have an increased risk of future fractures: a summary of the literature and statistical synthesis[J]. J Bone Miner Res, 2000, 15(4):721-739.

[2] Gunnes M, Mellstrom D, Johnell O. How well can a previous fracture indicate a new fracture? A questionnaire study of 29,802 postmenopausal women[J]. Acta Orthop Scand, 1998, 69(5):508512.

[3] Reed MR, Murray JR, Abdy SE, et al. The use of digital X-ray radiogrammetry and peripheral dual energy X-ray absorptiometry in patients attending fracture clinic after distal forearm fracture[J]. Bone, 2004, 34(4):716-719.

[4] Owen RA, Melton LJ 3rd, Ilstrup DM, et al. Colles' fracture and subsequent hip fracture risk[J]. Clin Orthop Relat Res, 1982, 171:37-43.

[5] Vogt MT, Cauley JA, Tomaino MM, Stone K, Williams JR, Herndon JH. Distal radius fractures in older women: a 10-year follow-up study of descriptive characteristics and risk factors. The study of osteoporotic fractures. J Am Geriatr Soc. 2002;50:97-103.

[6] Kelsey JL, Browner WS, Seeley DG, Nevitt MC, Cummings SR. Risk factors for fractures of the distal forearm and proximal humerus. The Study of Osteoporotic Fractures Research Group. American J Epidemiol. 1992;135:477-89.

[7] Mallmin H, Ljunghall S, Persson I, et al. Fractureof the distal forearm as a forecaster of subsequent hip fracture: a populationbased cohort study with 24 years of follow-up[J]. Calcif Tissue Int, 1993, 52:269-72.

[8] Cuddihy MT, Gabriel SE, Crowson CS, et al. Forearm fractures as predictors of subsequent osteoporotic fractures[J]. Osteoporos Int, 1999, 9(6):469-475.

[9] Freedman BA, Potter BK, Nesti LJ, et al. Missed opportunities in patients with osteoporosis and distal radius fractures[J]. Clin Orthop Relat Res, 2007, 454:202-6.

[10] Khan SA, de Geus C, Holroyd B, et al. Osteoporosis follow-up after wrist fractures following minor trauma[J]. Arch Intern Med, 2001, 161:1309-12.

[11] Gong HS, Oh WS, Chung MS, et al. Patients with wrist fractures are less likely to be evaluated and managed for osteoporosis[J]. J Bone Joint Surg Am, 2009, 91:2376-80.

[12] Barnett E, Nordin BE. The radiological diagnosis of osteoporosis: a new approach[J]. Clin Radiol, 1960, 11:166-174.

[13]Wilson J, Bonner TJ, Head M, et al. Variation in bone mineral density by anatomical site in patients with proximal humeral fractures[J]. J Bone Joint Surg Br, 2009, 91:772-

5.http://dx.doi.org/10.1302/0301-620X.91B6.22346. 
[14] Tingart MJ, Apreleva M, von Stechow D, et al. The cortical thickness of the proximal humeral diaphysis predicts bone mineral density of the proximal humerus[J]. J Bone Joint Surg $\mathrm{Br}$, 2003, 85:611-7.http://dx.doi.org/10.1302/0301620X.85B4.12843.

[15] Mather J, MacDermid JC, Faber KJ, et al. Proximal humerus cortical bone thickness correlates with bone mineral density and can clinically rule out osteoporosis[J]. J Shoulder Elbow Surg, 2013, 22(6):732-738.

[16] Tracy Webber, Shaun P.Patel, Michael Pensak, et al】 Correlation Between Distal Radial Cortical Thickness and Bone Mineral Density[J]. J Hand Surg Am, 2015, 493-499.

[17] Jannike Øyen, MSc, Christina Brudvik, PhD, MD, Clara Gram Gjesdal, et al. Osteoporosis as a Risk Factor for Distal Radial

Fractures: A case-control study [J]. J Bone Joint Surg Amr, 2011, 93:248-356.

[18] Papaioannou A, Santesso N, Morin SN, et al. Recommendations for preventing fracture in long $\triangle$ term care[J]. CMAJ, 2015, 187(15):1135囚1144, E450هE461. DOI: 10.1503/cmaj.141331.

[19] Cosman F, de Beur SJ, LeBoff MS, et al. Clinician's guide to prevention and treatment of osteoporosis[J]. Osteoporos Int, 2014, 25(10): 2359『2381. DOI: 10.1007/s00198ه014『2794『2.

[20] Kanis JA, Borgstrom F, de Laet C, et al『Assessment of fracture risk[J]区 Osteoporosis Int, 2005, 16: 581-589》

[21] Soichiro Itoh, Hideki Tomioka. Relationship between bone mineral density of the distal radius and ulna and fracture charcateristics. J The journal of hand surgery, 2004, 29:123-130.

[22] Kang D, Liu Z, Wang Y, et al《Xelationship of body composition with bone mineral density in northern Chinese men by body mass index levels[J]囚J Endocrinol Invest, 2014, 7(4):359367区

[23] Wee J, Song BY, Shen L, et al囚The relationship between body mass index and physical activity levels in relation to bone mineral density in premenopausal and postmenopausal women[J]区Arch Oste oporos, 2013, 8(2):162-165】

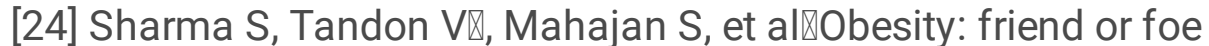
for osteoporosis[J]ญ Midlife Health, 2014, 5(1):6-9》

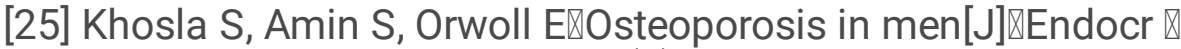
ev, 2008, 29(4):441区

[26] Rausch S, Klos K, Gras F, et al. Utility of the cortical thickness of the distal radius as a predictorof distal-radius bone density[J]. Arch Trauma Res, 2013, 2(1):11-15.

[27] Ward KA, Adams JE, Hangartner TNखRecommendations for Thresholds for Cortical Bone Geometryand Density Measurementby Peripheral Quantitative Computed Tomography[J]区Calcif Tissuelnt, 2005, 77:275-280》 
[28] Pistoia W, Van Reitbergen B, Rüegsegger $P \bigotimes$

Mechanicalconsequences of different scenarios for simulated bone atrophyand recovery in the distal radius[J]هBone, 2003, 33( 6):937-945》

[29] Hollevoet N, Verdonk R. Outcome of distal radius fractures inrelation to bone mineral density[J]. Acta Orthop Belg, 2003, 69(6):510-514.

[30] Feldstein AC, Nichols GA, Elmer PJ, et al. Older women with fractures: patients falling through thecracks of guidel-inerecommended osteoporosis screening and treatment[J]. J Bone Joint Surg Am, 2003, 85(12):2294-2302.

\section{Tables}

\begin{tabular}{|c|c|c|c|c|}
\hline \multirow[t]{2}{*}{ cohort } & osteoporotic & $\begin{array}{c}\text { non- } \\
\text { osteoporotic }\end{array}$ & $t$ & $\mathrm{P}$ \\
\hline & $\square \mathrm{n}=96 \square$ & $\square \mathrm{n}=51 \square$ & & \\
\hline age[year— & $66.8 \pm 9.4$ & $58.2 \pm 6.4$ & 6.584 & 0.000 \\
\hline weight $\square \mathrm{kg} \square$ & $55.8 \pm 8.1$ & $62.4 \pm 8.8$ & -4.517 & 0.000 \\
\hline $\mathrm{BMI} \square \mathrm{kg} / \mathrm{m} 2 \square$ & $23.4 \pm 4.0$ & $25.4 \pm 3.5$ & -2.991 & 0.003 \\
\hline DRCT $\square \mathrm{mm} \square$ & $4.22 \pm 1.01$ & $5.33 \pm 0.85$ & -7.009 & 0.000 \\
\hline ratio & $0.29 \pm 0.08$ & $0.35 \pm 0.07$ & -4.548 & 0.000 \\
\hline
\end{tabular}

Table2 Relationship between DRCT and BMD $\square$ cortex ratio and BMD by Pearson correlation analysis

\begin{tabular}{cccccc}
\hline Dependent variable & Independent variable & $\mathrm{r}$ & $\mathrm{P}$ & $\mathrm{R}^{2}$ & $\mathrm{P}$ \\
\hline DRCT & hip bone density & 0.393 & 0.000 & 0.154 & 0.000 \\
DRCT & lumbar bone density & 0.529 & 0.000 & 0.280 & 0.000 \\
Cortex ratio & hip bone density & 0.337 & 0.000 & 0.114 & 0.000 \\
Cortex ratio & lumbar bone density & 0.414 & 0.000 & 0.171 & 0.000 \\
\hline
\end{tabular}

\begin{tabular}{ccccc}
\hline \multicolumn{2}{c}{$\begin{array}{c}\text { Table3 } \\
\text { values for predicting osteoporosis }\end{array}$} & \multicolumn{2}{c}{$\begin{array}{c}\text { Sensitivity, } \\
\text { verificity }\end{array}$} & and NPV of DRCT threshold \\
\hline DRCT & Sensitivity & Specificity & NPV & Youden index \\
\hline $5.5 \mathrm{~mm}$ & $92.7 \%$ & $43.1 \%$ & $74.1 \%$ & 0.358 \\
$5.4 \mathrm{~mm}$ & $91.7 \%$ & $51.0 \%$ & $76.5 \%$ & 0.427 \\
$5.3 \mathrm{~mm}$ & $90.6 \%$ & $51.0 \%$ & $74.3 \%$ & 0.416 \\
$5.2 \mathrm{~mm}$ & $88.5 \%$ & $58.8 \%$ & $63.2 \%$ & 0.473 \\
$5.1 \mathrm{~mm}$ & $83.3 \%$ & $72.7 \%$ & $66.7 \%$ & 0.560 \\
$5.0 \mathrm{~mm}$ & $78.1 \%$ & $56.1 \%$ & $52.3 \%$ & 0.342 \\
\hline
\end{tabular}




\section{Figures}

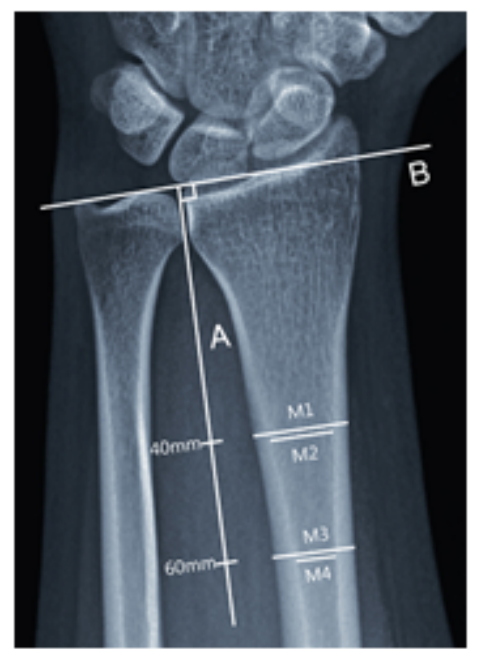

Figure 1

Illustration of DRCT measurement method. Beginning at the radial-most edge of the distal ulnar articular surface, a line was extended $40 \mathrm{~mm}$ proximally. At this level, the cortical width (M1) and width of the medullary canal (M2) were measured. Another set of measurements of cortical width (M3) and medullary canal width (M4) were performed $20 \mathrm{~mm}$ proximally. The DRCT is defined as $([\mathrm{M} 1-\mathrm{M} 2]+[\mathrm{M} 3-\mathrm{M} 4]) / 2$, and the cortex ratio is defined as DRCT/[(M1+M3)/2].

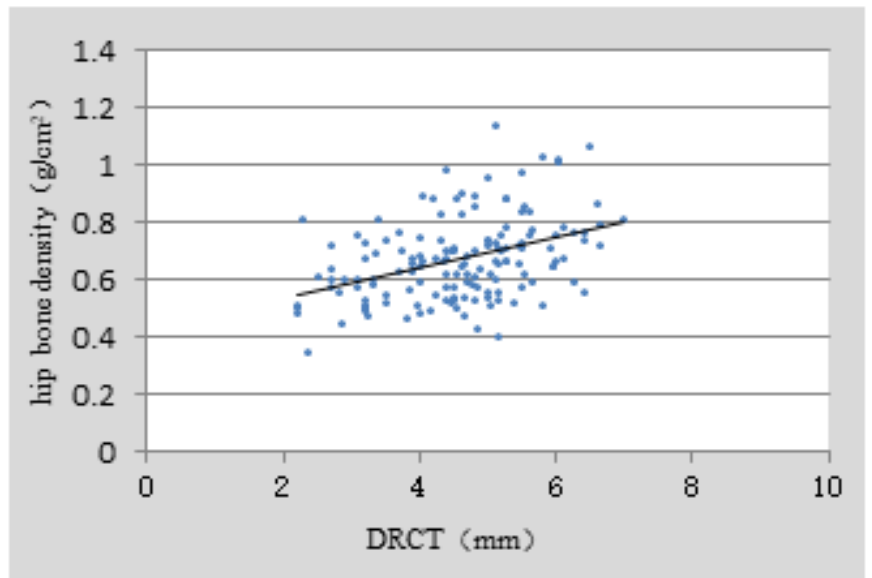

Figure 2

Scatterplot showing a positive correlation between hip BMD and DRCT. 


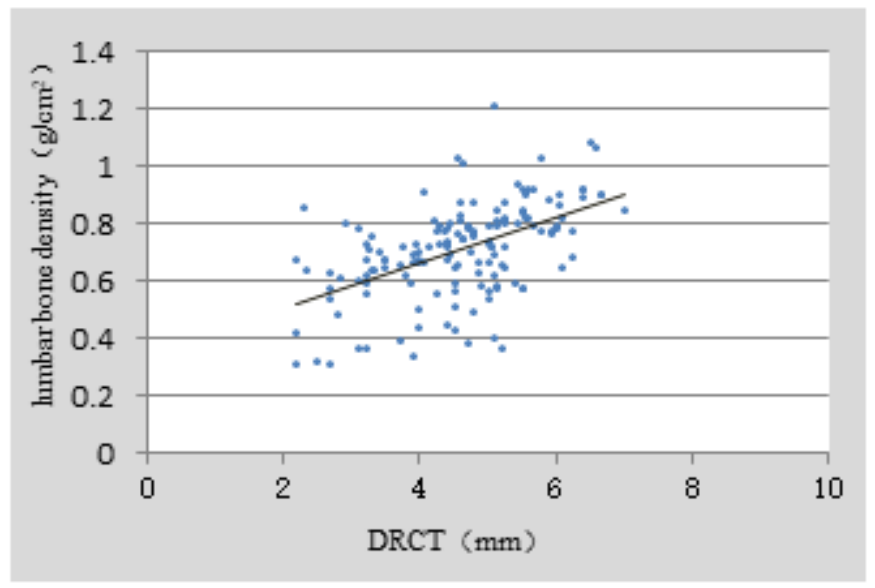

Figure 3

Scatterplot showing a positive correlation between lumbar spine BMD and DRCT.

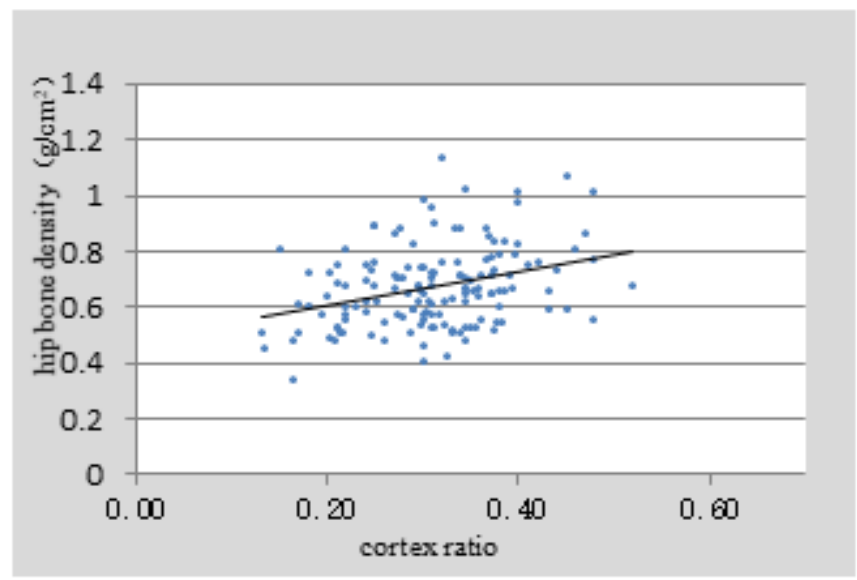

Figure 4

Scatterplot showing a positive correlation between hip BMD and the cortex ratio.

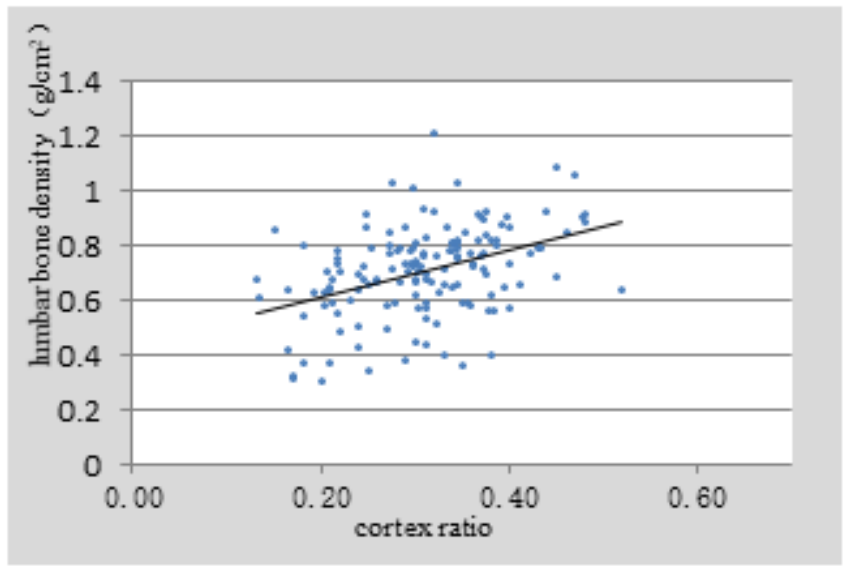

Figure 5

Page 13/14 
Scatterplot showing a positive correlation between lumbar spine $\mathrm{BMD}$ and the cortex ratio.

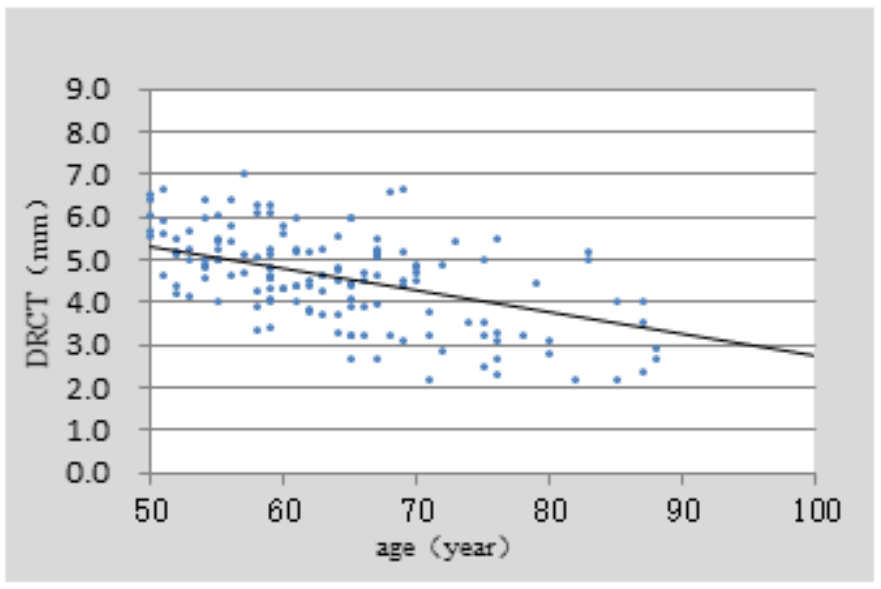

Figure 6

Scatterplot showing a negative correlation between DRCT and age.

\section{Supplementary Files}

This is a list of supplementary files associated with this preprint. Click to download.

Data.xls 